\title{
An Environment for Runtime Power Monitoring Of Wireless Sensor Network Platforms
}

\author{
Aleksandar Milenkovic, Milena Milenkovic, \\ Emil Jovanov, Dennis Hite \\ Electrical and Computer Engineering Department \\ The University of Alabama in Huntsville \\ Huntsville, AL 35899 USA \\ \{milenka | milenkm | jovanov | hitedw\}@ece.uah.edu
}

\author{
Dejan Raskovic \\ Electrical and Computer Engineering Department \\ University of Alaska Fairbanks \\ Fairbanks, AK 99775-5915 \\ d.raskovic@uaf.edu
}

Key Words: Power Consumption, Wireless Sensor Networks, Real-time Monitoring, Measurements

\begin{abstract}
Wireless sensor networks emerged as a key technology for prolonged, unsupervised monitoring in a wide spectrum of applications, from biological and environmental to civil and military. The sensor networks should operate autonomously for a long period of time with stringent resource and energy constraints. Energy conservation and power-awareness have become a focus of a number of research efforts, as sensor network nodes must operate on batteries or use energy extracted from the environment, such as solar energy or vibrations. Runtime power measurements and characterization of real existing systems are crucial for studies that target power optimizations, including techniques for dynamic adaptation based on the current energy status. This paper introduces an environment for unobtrusive real-time power monitoring that could be used for a number of wireless sensor platforms. We describe our methodology for calibration and validation of the environment and give empirical data for the Telos wireless sensor platform when it runs a subset of representative applications.
\end{abstract}

\section{INTRODUCTION}

Recent technology advances in sensors, microprocessors, and wireless communications have enabled design and proliferation of ad-hoc wireless sensor networks. These networks consist of a large number of inexpensive and miniature sensors that can monitor and control environments without human intervention for a long period of time. Current research efforts aim at developing of new sensing devices, architectures for sensor platforms [1] [2], wireless communication protocols [3] [4] [5], as well as system support for design and evaluation of sensor networks [6] [7].

Energy consumption is a first class design constraint in wireless sensor networks as they are typically battery operated. To extend each node's lifetime it is necessary to reduce power dissipation as much as possible; dissipation below 100 microwatts will enable operation on energy scavenged from the environment. Various design trade-offs between communication and on-sensor computation, collaborative protocols, and hierarchical network organization can yield significant energy savings. Once the sensor network is deployed dynamic power management techniques can be employed in order to maximize battery lifetime [8].

Wireless sensor network designers need a toolbox that will allow them to perform fast and accurate assessment of various design alternatives and trade-offs between design parameters, such as performance, total power consumption, reliability, and system lifetime. One approach to this challenge is to rely on full system simulators; however, they are not readily available, suffer from inaccuracies, and require long simulation time. In addition, designers often need the ability to measure live, running systems, and correlate measured data with overall hardware and software behavior. Power measurements in lab setup often fail to capture the pattern of power load of sensor nodes after they are deployed in real environments.

In this paper we present an inexpensive environment for unobtrusive power measurements for wireless sensor network platforms and describe its calibration and validation. The setup utilizes a clamp-on current probe rather than a commonly used shunt resistor. We have developed several illustrative TinyOS applications and collected their power traces for the Telos platform [9] that features a recently introduced IEEE 802.15.4-compliant wireless transceiver [10] and an ultra-low power microcontroller MSP430 [11]. In addition to power optimization and application tuning, the collected power traces can be used as an input for mathematical models designed for fast approximations of overall power consumption, or for initialization and calibration of more precise power simulators for wireless sensor networks, such as PowerTOSSIM [6].

The rest of the paper is organized as follows. Section 2 describes common techniques used for power measurements. Section 3 presents our setup for unobtrusive power measurements and its calibration. Section 4 presents power traces for several characteristic TinyOS applications running on a Telos A wireless sensor platform. Section 5 describes future work and concludes the paper. 


\section{POWER MEASUREMENTS}

Power consumption of a system under test can be determined in two ways (Fig. 1): using a clamp-on current probe or a shunt resistor. With the first approach we sample the power supply and the output voltage from the current probe, which is usually a linear function of current through the clamp (1). With the second approach we sample the power supply and the voltage at the shunt resistor, and the total power consumption of the system under test can be calculated as in (2). While measurements with current probes are unobtrusive, shunt resistors interfere with operation of the system under test and are unsuitable when there are large variations of current.

$$
\begin{aligned}
& P_{S U T}=V_{S U T} \cdot I=V_{S U P P L Y} \cdot I, \quad I=f\left(V_{C P R O B E}\right) \\
& P_{S U T}=V_{S U T} \cdot I=\left(V_{S U P P L Y}-V_{S H U N T}\right) \cdot \frac{V_{S H U N T}}{R_{S H U N T}}
\end{aligned}
$$

It should be noted that cycle-accurate energy characterization can be done using a recently proposed measurement system based on charge transfer [12]. However, this approach is rather expensive in time and cost, yet this level of precision is not necessary for determining power profiles.
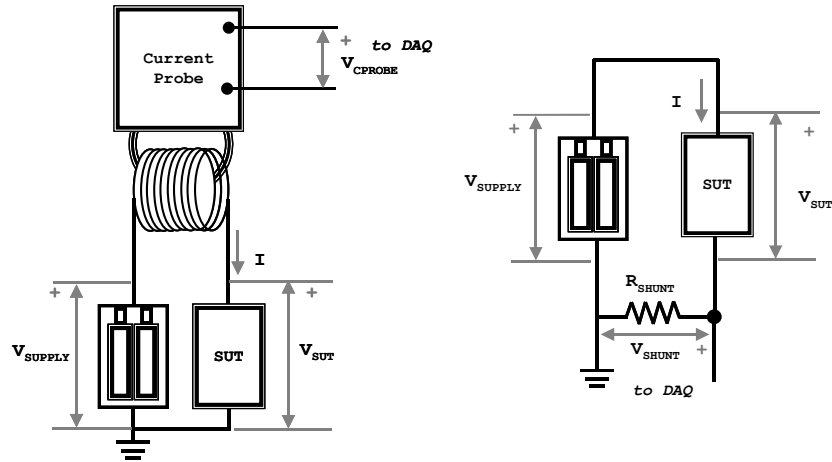

Fig. 1. Power measurements with current probe (left) and shunt resistor (right); SUT - System Under Test.

\section{SETUP FOR POWER MEASUREMENTS}

The block diagram of our environment for run-time power measurements is shown in Fig. 2. The environment consists of the following subblocks:

- The system under test - a wireless sensor platform and an energy source;

- The data acquisition subblock - a current probe for unobtrusive current measurements, a signal conditioning circuit, and a data acquisition card;

- The validation and calibration subblock - a digital multimeter;

- The logger laptop computer for data logging, processing, and inspection.

Fig. 3 shows a photo of our power measurement setup. To measure current through the power line we use an ExTech
380946 current probe. The output voltage from the current probe is connected to an Agilent 34401A digital multimeter (DMM), and a National Instrument DAQCard-AI-16XE-50 data acquisition card. Both voltage from the battery and voltage from the current probe are sampled with up to $200 \mathrm{~K}$ samples per second. A LabView application running on the logger laptop collects voltage data from the current clamp and voltage data from the battery. The data are stored and later processed in MATLAB.

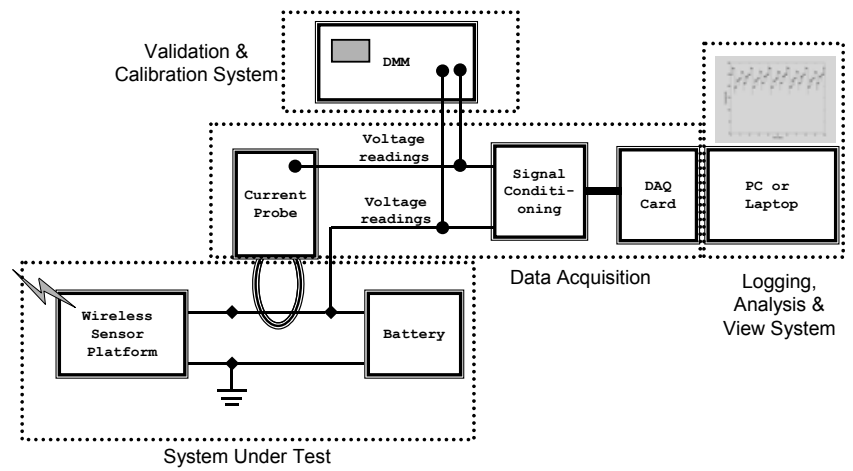

Fig. 2 Block diagram of the environment for run-time power monitoring for wireless sensor platforms. Description: DMM - Digital Multimeter, DAQ Data Acquisition Card.

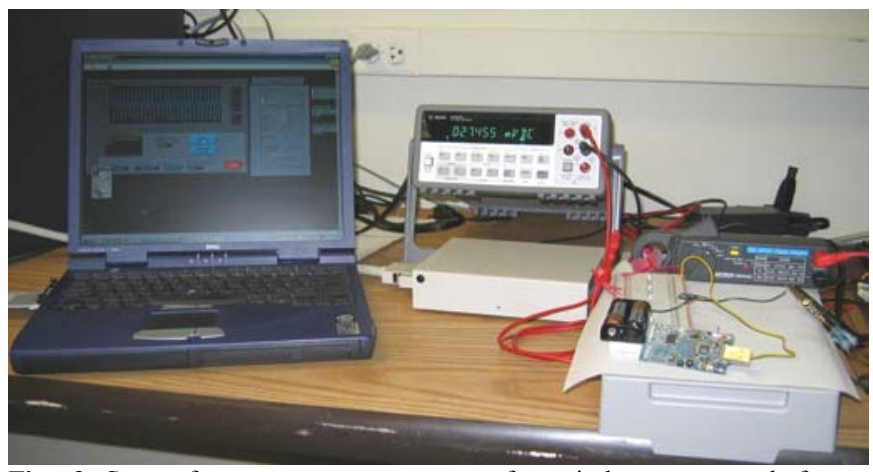

Fig. 3 Setup for power measurements for wireless sensor platforms. Description: Telos platform, 2xAA batteries, Current clamp ExTech 380946, Agilent 34401A Digital Multimeter, Shielded Connector Block SCB-68, National Instruments DAQCard-AI-16XE-50, LabView running on a laptop computer.

The current probe is clamped around the power supply line and outputs the voltage directly proportional to the measured current. The current probe sensitivity is $1 \mathrm{mV}$ output voltage for $1 \mathrm{~mA}$ of current. To increase the sensitivity of the current probe we made a solenoid with 10 rings of the power supply line. We selected this solenoid since the expected current is in range $0-40 \mathrm{~mA}$, and the smallest probe range is $0-400 \mathrm{~mA}$. The setup is calibrated using a simple circuit with the power supply and a resistor. We measured both the current $\mathrm{I}_{\mathrm{TEST}}$ and the power supply $\mathrm{V}_{\text {TEST }}$ for a range of resistors with resistances from $70 \Omega$ to $3 \mathrm{~K} \Omega$ using the digital multimeter. The results are shown in Fig. 4. Though we confirmed expected linear dependency, we found that it could be better approximated with a second order polynomial, since the 
mean squared deviation with the polynomial is 3.26 times less than with the linear function. Current in milliamps is calculated from voltage in volts as shown in (3).

$$
I=-20 \cdot V_{C P R O B E}^{2}+99.1 \cdot V_{C P R O B E}+0.1576
$$

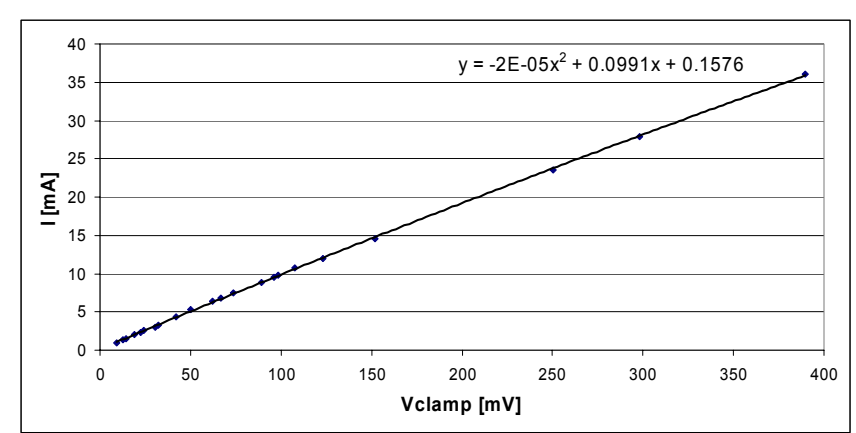

Fig. 4. Current probe calibration.

The selected current probe is susceptible to noise. The output voltage of the idle current probe with open solenoid ends is in the range of -7.41 to $7.44 \mathrm{mV}$, with the mean value of $0.36 \mathrm{mV}$ and standard deviation of $1.55 \mathrm{mV}$ (Fig. 5), with the sampling frequency of $100 \mathrm{Ksamples} / \mathrm{sec}$. Spectral analysis of the noise shows distinct frequency components close to $5+10 \mathrm{i} \mathrm{KHz}, \mathrm{i}=0,1,2,3,4$ (Fig. 6). We verified whether these components come from aliasing high frequencies, by applying a low-pass RC filter with $50 \mathrm{KHz}$ cut-off frequency on the current probe output. The spectrum of noise was not changed with this filter. This noise can be reduced with a low-pass $\mathrm{RC}$ filter, but at the price of flattening the real signal.

All power traces are collected with sampling frequency of $200 \mathrm{Ksamples} / \mathrm{sec}$ and they are compared to the corresponding traces collected using a shunt resistor. In general, the shunt resistor traces suffer less from the noise, but the trace collection is not unobtrusive.

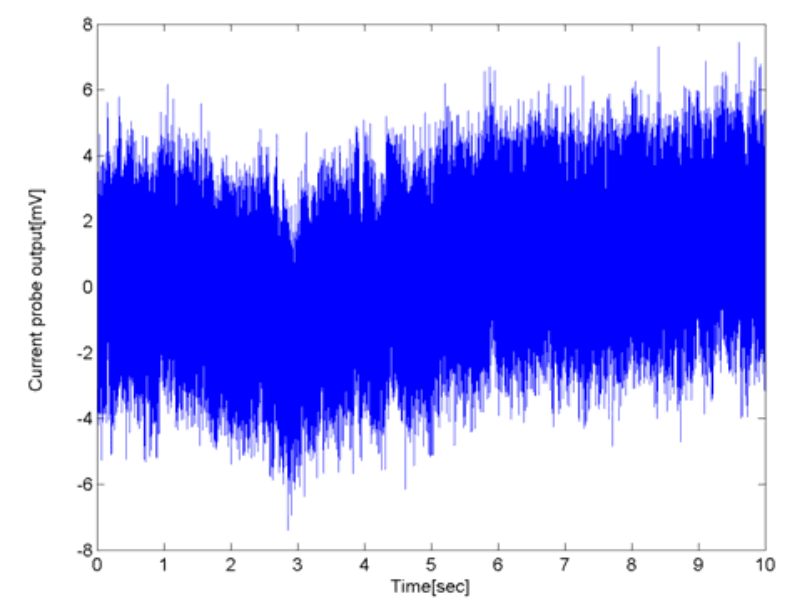

Fig. 5. Output voltage from the current probe with open solenoid ends. Sampling rate is $100 \mathrm{KSamples} / \mathrm{s}$.

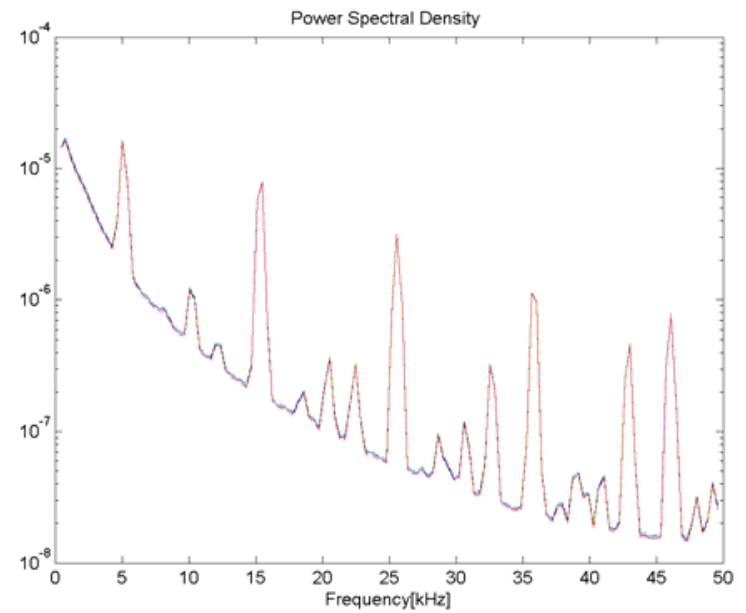

Fig. 6. Power spectral density of noise.

\section{RESULTS}

Power measurements are performed using Telos, a recently introduced wireless sensor platform designed for low-power operation, ease of use, and hardware and software robustness [9]. Telos is powered by two AA batteries and features a Chipcon 2420 radio in the $2.4 \mathrm{GHz}$ band [10]; an $8 \mathrm{MHz}$ Texas Instruments MSP430 microcontroller [11]; an integrated onboard antenna with $50 \mathrm{~m}$ range indoors / $125 \mathrm{~m}$ range outdoors; a USB port for programming and communication; an external flash memory; and integrated humidity, temperature, and light sensors. The MSP430 microcontroller is based around a 16-bit RISC core integrated with RAM and flash memories, analog and digital peripherals and flexible clock subsystem. It supports several low-power operating modes and consumes as low as $1 \mu \mathrm{A}$ in a standby mode; it also has very fast wake up time of no more than $6 \mu$ s. Telos Revision A features a MS430F149 microcontroller with $2 \mathrm{~KB}$ RAM and $60 \mathrm{~KB}$ flash memory; Telos Revision B features a MSP430F1611 with $10 \mathrm{~KB}$ of RAM and $48 \mathrm{~KB}$ of flash memory. The CC2240 wireless transceiver is IEEE 802.15.4 compliant; it has programmable output power, maximum data rate of $250 \mathrm{Kbs}$, and hardware support for error correction and encryption. The CC2240 is controlled by the MSP430 microcontroller through the SPI port and a series of digital $\mathrm{I} / \mathrm{O}$ lines with interrupt capabilities.

To demonstrate characteristic behaviors of wireless sensor platforms we have collected power traces for several TinyOS applications [13]. First, we measured CntToLedsAndRfm, which increments an internal counter variable with a $4 \mathrm{~Hz}$ frequency. On each counter tick, the least significant 3 bits of the counter are displayed on the Telos LEDs and the radio transmits the entire 16-bit counter value. Fig. 7 shows a 10second power trace for CntToLedsAndRfm. The power trace clearly indicates repeating 2 -second sequences, each cycling through the counter sequence $0-7$ and radio transmission. 


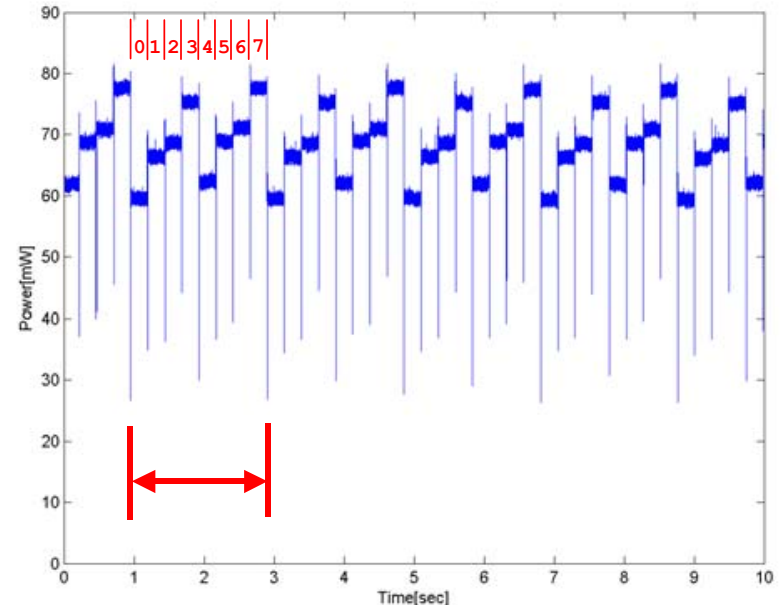

Fig. 7. Power traces the CntToLedsAndRfm application under TinyOS running on a Telos A platform.

To illustrate low-power operating modes and breakdown of power consumption for the radio and the MSP430 we have developed a TinyOS application named Testera. In this application a Timer event is triggered every $125 \mathrm{~ms}$. In the Timer interrupt routine a 16-bit counter variable $C n t$ is incremented by modulo 1024. The current value of $\mathrm{Cnt}$ is placed in a buffer that keeps 10 most recent counter values. When the buffer is full a message is prepared (26 byte payload) and sent over the radio. A power trace of the Telos A platform when running Testera for $5 \mathrm{~s}$ is shown in Fig. 8. In this trace we can clearly identify small peaks when the MSP430 is waken up to update the Cnt counter; a magnified power trace of $5 \mathrm{~ms}$ is shown in the left box in Fig. 8. Every $10^{\text {th }}$ counter update involves an RF message transmission; a magnified power trace of $10 \mathrm{~ms}$ is shown in the right box (Fig. 8). The total power consumption is nearly constant around $65 \mathrm{~mW}$ and is predominantly determined by power consumed by the radio. The $\mathrm{C} 2420$ radio draws $20 \mathrm{~mA}$ in the receive mode and $17.4 \mathrm{~mA}$ in the transmit mode. The power trace allows us to clearly identify different phases of the application execution, such as the count up, communication between the MSP430 and the CC2420 over the SPI port, and the very message transmission.

Application TesteraRadioOnOff is built upon Testera and utilizes Split Radio Control interface. This interface allows programmable control over the radio. The radio is turned on only when a radio packet is ready to be sent. When the message buffer is full, instead of an immediate sending of the message, a request for RadioOn is initiated. When we receive confirmation that the radio is on and ready to receive a new message, the message is sent. Upon receiving confirmation that the message has been sent, the radio is turned off. A power trace for this application is shown in Fig. 9. The total power consumption averages around $5 \mathrm{~mW}$ with peaks around $9 \mathrm{~mW}$ in timer interrupt routines. The left block in Fig. 9 shows a magnified power trace of $5 \mathrm{~ms}$ for the count up event. The right block in Fig. 9 shows a magnified power trace of $40 \mathrm{~ms}$ during packet radio transmission. The whole event when the buffer is full now encompasses several steps that precede radio transmission. It takes approximately $6 \mathrm{~ms}$ to start the radio, then the MSP430 sends the message over the SPI, and finally the message is transmitted over the radio.

All measurements are repeated with the setup with shunt resistors. We evaluated several configurations with $R_{S}=0.5 \Omega$ and $R_{S}=4.8 \Omega$. The traces collected on the shunt resistors exhibit the same trends as the traces collected with the current probe. However, the actual values of samples are lower due to presence of the shunt resistor. Fig. 10 shows a power trace for the Testera application taken with $\mathrm{R}_{\mathrm{S}}=4.8 \Omega$.

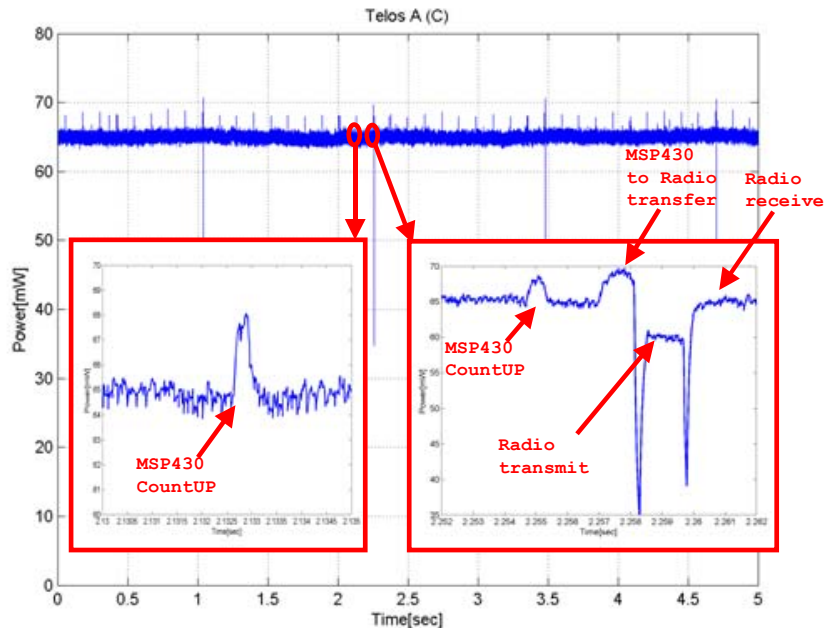

Fig. 8 Power traces for the Testera TinyOS application running on Telos A.

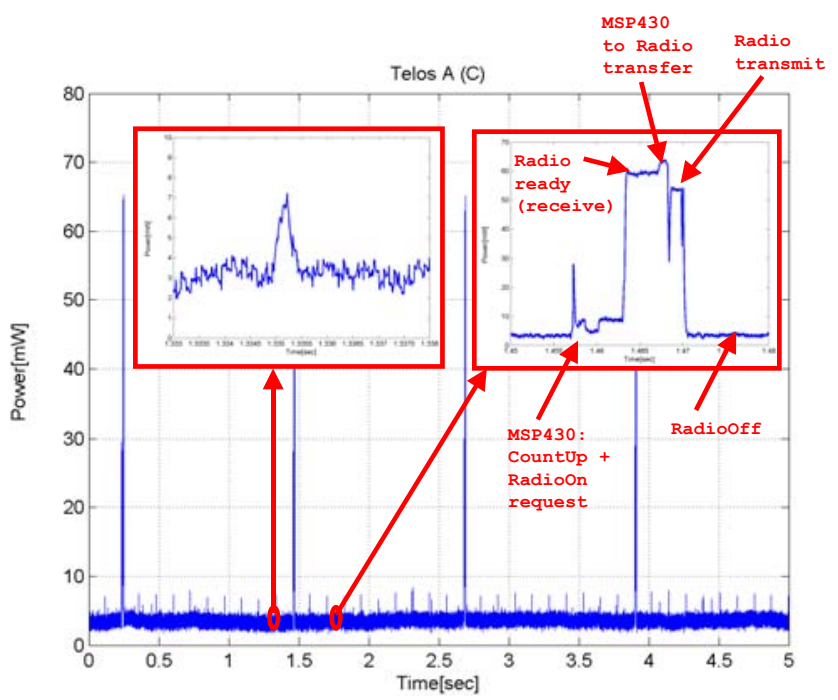

Fig. 9. Power traces for the TesteraRadioOnOff TinyOS application running on Telos A. 


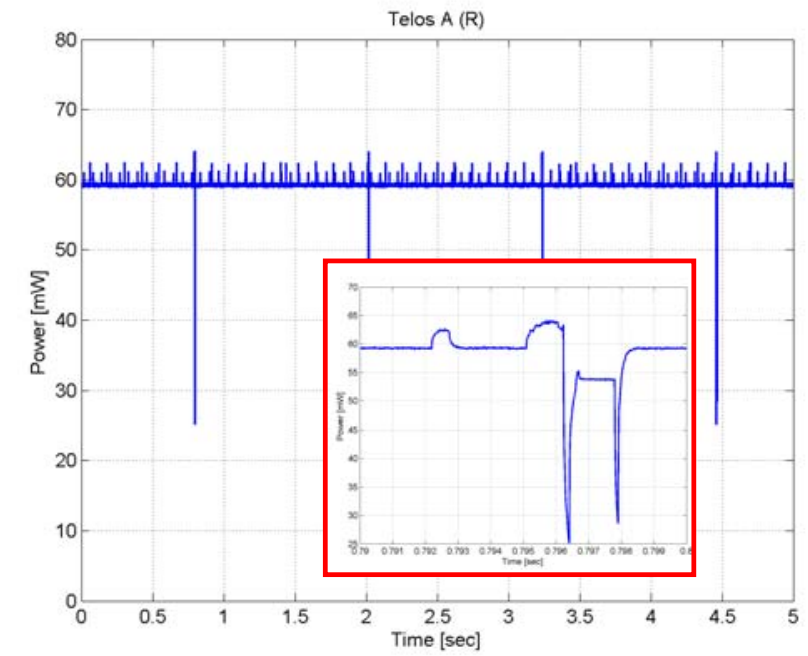

Fig. 10. Power traces for the Testera TinyOS application running on Telos A measured by the shunt resistor.

\section{CONCLUSIONS}

The primary contributions of this paper are as follows:

- We describe an environment for collection and processing of runtime power traces for wireless sensor platforms.

- We describe verification and calibration of the environment. Once verified and calibrated, the setup can be easily used for live measurements of deployed wireless sensor networks, to help fine-tuning and power optimizations.

- We present runtime total power measurements for the Telos platform and characterize power consumption for several typical operating modes.

We plan to use this setup for various research efforts targeting energy-efficient sensor networks. In particular, we plan to use it for after-deployment tracing in order to capture sensors behavior in real environments, where temperature, supply voltage, and type of responses are likely to vary significantly in time. The existing set of applications can also be extended with new microbenchmarks that will capture power traces when multiple physical signals are sensed (AD converter), stored locally in the external flash memory, and later transmitted. Various design trade-offs can be evaluated, for example, effects of data compression and encryption.

\section{ACKNOWLEDGMENTS}

This work is being supported in part by National Science Foundation grant IIS-0434156 and the University of Alabama in Huntsville.

\section{REFERENCES}

[1] J. Hill, R. Szewcyk, A. Woo, D. Culler, S. Hollar, and K. Pister, "System Architecture Directions for Networked Sensors," in Proceedings of the 9th ASPLOS, Cambridge, MA, 2000, pp. 93 -- 104. J. Hill and D. Culler, "MICA: A Wireless Platform for Deeply Embedded Networks," IEEE Micro, vol. 22, November 2002, pp. 12 -- 24.

[3] J. M. Rabaey, M. J. Ammer, J. L. d. S. Jr., D. Patel, and S. Roundy, "PicoRadio Supports Ad Hoc UltraLow Power Wireless Networking," IEEE Computer, vol. 33, July 2000, pp. 42 - 48.

[4] S. Roundy, B. Otis, Y.-H. Chee, J. Rabaey, and P. Wright, "A 1.9GHz RF Transmit Beacon Using Environmentally Scavenged Energy," in Digest IEEE Int. Symposium on Low Power Elec. and Devices, Seoul, Korea, 2003, pp. -.

[6] V. Shnayder, M. Hempstead, B.-r. Chen, G. W. Allen, and M. Welsh, "Simulating the Power Consumption of Large-Scale Sensor Network Application," in Proceedings of the Second ACM Conference on Embedded Networked Sensor Systems (SenSys'04), Baltimore, MD, USA, 2004, pp. 188 -- 200.

[7] P. Levis, N. Lee, M. Welsh, and D. Culler, "TOSSIM: Accurate and Scalable Simulation of Entire TinyOS Applications," in First ACM Conference on Embedded Networked Sensor Systems (SenSys 2003), Los Angelos, California, USA, 2003, pp. 126 - 137.

[8] A. Sinha and A. Chandrakasan, "Dynamic Power Management in Wireless Sensor Networks," IEEE Design \& Test of Computers, vol. 18, March 2001 2001, pp. 62 -- 74.

[9] J. Polastre, R. Szewczyk, and D. Culler, "Telos: Enabling Ultra-low Power Wireless Research", $<$ http://www.moteiv.com>

--, "Chipcon RF Tranceivers", <www.chipcon.com> --, "MSP430 MCUs", <www.ti.com/msp430> (January 2004).

N. Chang, K. Kim, and H. G. Lee, "Cycle-accurate Energy Measurement and Characterization With a Case Study of the ARM7TDMI," IEEE Transactions on Very Large Scale Integration (VLSI) Systems, vol. 10, April 2002, pp. 146 -- 154. --, "TinyOS", <http://www.tinyos.net/> 